\title{
Correlated Analytic Hierarchy Process
}

\author{
Hsiang-Hsi Liu, ${ }^{1}$ Yeong-Yuh Yeh, ${ }^{2}$ and Jih-Jeng Huang ${ }^{3}$ \\ ${ }^{1}$ Graduate Institute of International Business, National Taipei University, Sanxia, Taiwan \\ ${ }^{2}$ Department of Business Management, Aletheia University, New Taipei City, Taiwan \\ ${ }^{3}$ Department of Computer Science \& Information Management, Soochow University, Taipei, Taiwan
}

Correspondence should be addressed to Jih-Jeng Huang; jjhuang@scu.edu.tw

Received 8 May 2014; Accepted 29 July 2014; Published 18 August 2014

Academic Editor: Tofigh Allahviranloo

Copyright (C) 2014 Hsiang-Hsi Liu et al. This is an open access article distributed under the Creative Commons Attribution License, which permits unrestricted use, distribution, and reproduction in any medium, provided the original work is properly cited.

\begin{abstract}
The analytic hierarchy process (AHP) has been the most popular tool for the field of decision making in the past 30 years, because of its simplicity and rationality. Construct a hierarchy system for evaluation by decision makers. Hence, only the effect of outerdependence can be considered in the AHP. However, besides outer-dependence, correlation is another common effect between criteria which cannot be accounted for neither by the AHP nor by the analytic network process (ANP). Hence, in this paper, we extend the AHP to consider the correlation effect. In addition, a biobjective programming model is proposed to derive the result. Furthermore, the traditional AHP can be considered as the special case of the proposed model when the correlation effect between criteria is ignored. Finally, a numerical example is given to justify the proposed method and compare the result with the AHP.
\end{abstract}

\section{Introduction}

Since Bernoulli [1] proposed the concept of utility function to reflect human persuading, such as maximum satisfactory, and von Neumann and Morgenstern [2] presented the theory of game and economic behavior model, which expanded the studies on human being economic behavior for multiple attribute decision making (MADM) problems, from that moment on, more and more literatures engaged in this field. Roughly speaking, the procedures of MADM can be comprised of five main steps as follows [3].

Step 1. Define the nature of problem.

Step 2. Construct a hierarchy system for evaluation (as shown in Figure 1).

Step 3. Select the appropriate evaluating model.

Step 4. Obtain the relative weights and performance score of each attribute with respect to each alternative.

Step 5. Determine the best alternative according to the synthetic utility values, which are the aggregation value of relative weights and performance scores corresponding to alternatives.

On the basis of dealing with MADM problems, the analytic hierarchy process (AHP) was proposed by Saaty $[4,5]$ to derive the relative weights according to the appropriate hierarchical system. The AHP should be the most popular method used in dealing with multicriteria decision making (MCDM) problems in the field of operations research/management science (OR/MS), according to the published journal papers. To extend the capability of the AHP for dealing with more sophisticated problems, many methods have been proposed to revise or modify the limitations and defects of the AHP. For example, Yu [6] proposed a revised model to release the assumption of reciprocal matrix in the AHP and Huang [7] extended the AHP to consider the fuzzy environments.

Although the past papers elaborated much work on the extension of the AHP, they seem to ignore the issue of correlation between criteria. As we know, the AHP is used to derive the weights of criteria when they are independent and outerdependent by the upper-level criteria. However, there are other effects, such as inner-dependence and feedback, which should be considered to present the relationship between 


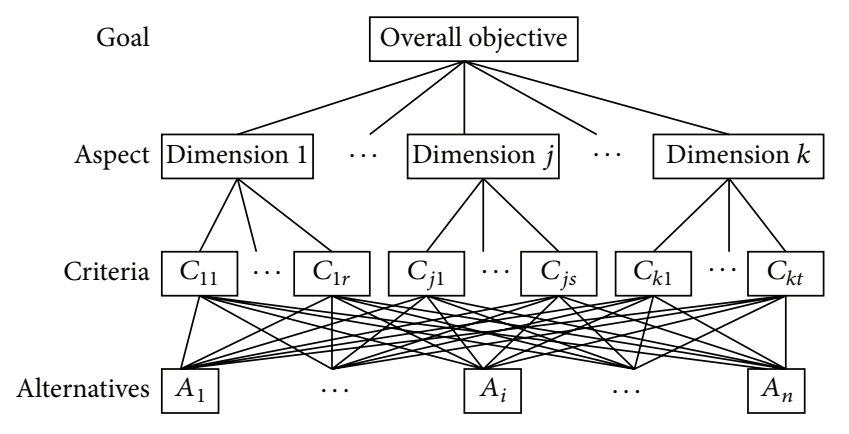

Figure 1: Hierarchical system for MADM.

criteria. Hence, Saaty [8] proposed the analytic network process to account for the effects of outer-dependence, innerdependence, and feedback. Hereafter, the AHP/ANP is the most common tool in the field of decision making to deal with various effects between criteria.

The correlation between criteria is commonly observed in the realistic problems. Let us consider the following investment situation to describe the insufficiency of the AHP/ANP as follows. Assume an investor is considering constructing a portfolio in a stock market. Two major criteria, that is, risk and return, are considered and can be divided into many independent subcriteria, respectively. The problem above can be solved by the AHP, because of the independent subcriteria. However, it is irrational to assume that risk and return are independent of each other in the real world. In addition, there is no evidence that risk can affect return and vice visa. All we can say is that risk and return are correlated with each other. Hence, the AHP/ANP cannot be used in this situation.

The purpose of this paper is to propose the correlated analytic hierarchy process (CAHP) which can account for the correlation between criteria in the AHP. A biobjective programming model is proposed to derive the result. In addition, we propose a numerical example to demonstrate the proposed model and compare the result with the traditional AHP. It should be highlighted that the tradition AHP can be considered as the special case when we reduce our model to single-objective programming model.

The rest of this paper is organized as follows. In Section 2, we present the procedure of the AHP and uncover the limitation on the issue of correlation between criteria. The way to develop the CAHP is given in Section 3. An application used here to demonstrate the proposed method is in Section 4. Discussions are presented in Section 5 and conclusions are in the last section.

\section{Analytic Hierarchy Process}

Analytic hierarchy process was proposed by Saaty $[4,5]$ to model subjective decision making processes based on multiple attributes in a hierarchical system. From that moment on, it has been widely used in corporate planning, portfolio selection, and benefit/cost analysis by government agencies for resource allocation purposes. It should be highlighted

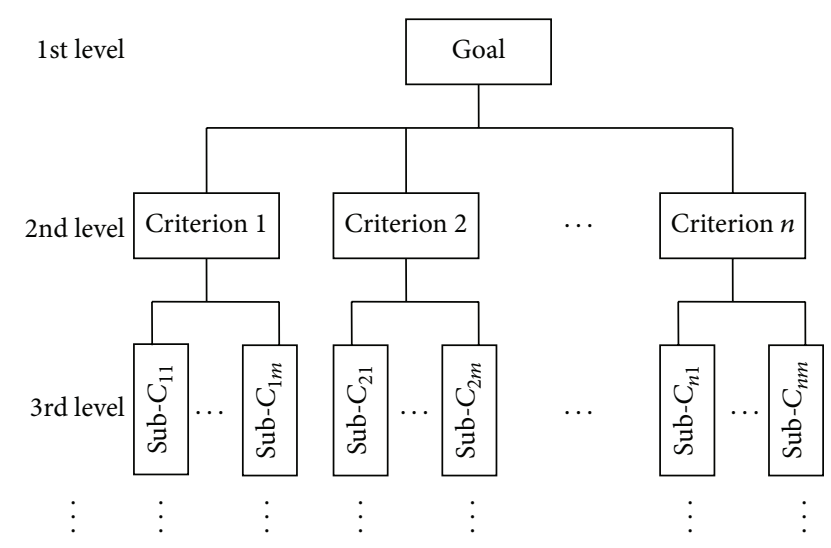

FIgURE 2: The hierarchical structure of the AHP.

that all decision problems are considered as a hierarchical structure in the AHP. The first level indicates the goal for the specific decision problem. In the second level, the goal is decomposed of several criteria and the lower levels can follow this principal to divide into other subcriteria. Therefore, the general form of AHP can be depicted as shown in Figure 2.

The main four steps of the AHP can be summarized as follows.

Step 1. Set up the hierarchical system by decomposing the problem into a hierarchy of interrelated elements/criteria.

Step 2. Compare the comparative weight between the attributes of the decision elements to form the reciprocal matrix.

Step 3. Synthesize the individual subjective judgment and estimate the relative weight.

Step 4. Aggregate the relative weights of the elements to determine the best alternatives/strategies.

In this section, the method of the AHP is firstly reviewed as follows. If we wish to compare a set of $n$ attributes pairwise according to their relative weights (importance), where the weights are denoted by $w_{1}, w_{2}, \ldots, w_{n}$, then the matrix of weight ratios can be represented as

$$
\mathbf{W}=\left[w_{i j}\right]_{n \times n},
$$

where $w_{i j}=w_{j i}^{-1}, w_{i j}=w_{i k} w_{k j}$, and $w_{i j}=w_{i} / w_{j}$.

Multiplying $\mathbf{W}$ by the weight vector, $\mathbf{w}$, yields

$$
\mathbf{W w}=\left[\begin{array}{ccccc}
\frac{w_{1}}{w_{1}} & \cdots & \frac{w_{1}}{w_{j}} & \cdots & \frac{w_{1}}{w_{n}} \\
\vdots & & \vdots & & \vdots \\
\frac{w_{i}}{w_{1}} & \cdots & \frac{w_{i}}{w_{j}} & \cdots & \frac{w_{i}}{w_{n}} \\
\vdots & & \vdots & & \vdots \\
\frac{w_{n}}{w_{1}} & \cdots & \frac{w_{n}}{w_{j}} & \cdots & \frac{w_{n}}{w_{n}}
\end{array}\right]\left[\begin{array}{c}
w_{1} \\
\vdots \\
w_{j} \\
\vdots \\
w_{n}
\end{array}\right]=n\left[\begin{array}{c}
w_{1} \\
\vdots \\
w_{j} \\
\vdots \\
w_{n}
\end{array}\right]=n \mathbf{w}
$$


or

$$
(\mathbf{W}-n \mathbf{I}) \mathbf{w}=\mathbf{0} .
$$

Next, in order to estimate the weight ratio $w_{i j}$ by $a_{i j}$, where $\mathbf{A}=\left[a_{i j}\right]_{n \times n}$, we can calculate the approximate weights by finding the eigenvector $\mathbf{w}$ with respect to $\lambda_{\max }$ which satisfies

$$
\mathbf{A w}=\lambda_{\max } \mathbf{w},
$$

where $\lambda_{\max }$ is the largest eigenvalue of the matrix $\mathbf{A}$. In addition, since $\mathbf{A}$ is an approximate for $\mathbf{W}$, we should calculate the consistency indexes (C.I.) to check if the consistency condition is almost satisfied for $\mathbf{A}$ using the following equation:

$$
\text { C.I. }=\frac{\lambda_{\max }-n}{n-1}
$$

where $\lambda_{\max }$ is the largest eigenvalue and $n$ denotes the numbers of the attributes. Saaty [5] suggested that the value of the C.I. should not exceed 0.1 for a confident result.

On the other hand, for the AHP, a near consistent matrix A with a small reciprocal multiplicative perturbation of a consistent matrix is given by [9]

$$
\mathbf{A}=\mathbf{W} \cdot \mathbf{E},
$$

where - denotes the Hadamard product, $\mathbf{W}=\left[w_{i j}\right]_{n \times n}$ is the matrix of weight ratios, and $\mathbf{E} \equiv\left[\varepsilon_{i j}\right]_{n \times n}$ is the perturbation matrix, where $\varepsilon_{i j}=\varepsilon_{j i}^{-1}$.

From (4) and (6), it can be seen that

$$
\begin{gathered}
\sum_{j=1}^{n} a_{i j} w_{j}-\lambda_{\max } w_{i}=0, \\
\lambda_{\max }=\sum_{j=1}^{n} a_{i j} \frac{w_{j}}{w_{i}}=\sum_{j=1}^{n} \varepsilon_{i j} .
\end{gathered}
$$

On the other hand, the multiplicative perturbation can be transformed to an additive perturbation of a consistent matrix such that

$$
\sum_{j=1}^{n} \frac{w_{i}}{w_{j}} \varepsilon_{i j}=\sum_{j=1}^{n} \frac{w_{i}}{w_{j}}+v_{i j},
$$

where $v_{i j}$ is the additive perturbation.

Since $\sum_{j=1}^{n} a_{i j} w_{j} / w_{i}=\sum_{j=1}^{n} \varepsilon_{i j}$, we can rewrite (8) as

$$
\begin{gathered}
\sum_{j=1}^{n}\left(\frac{w_{i}}{w_{j}} a_{i j} \frac{w_{j}}{w_{i}}\right)=\sum_{j=1}^{n}\left(\frac{w_{i}}{w_{j}} \varepsilon_{i j}\right)=\sum_{j=1}^{n}\left(\frac{w_{i}}{w_{j}}+v_{i j}\right), \\
\sum_{j=1}^{n} v_{i j}=\sum_{j=1}^{n}\left(a_{i j}-\frac{w_{i}}{w_{j}}\right) .
\end{gathered}
$$

On the basis of (8)-(10), it can be seen that $\lambda_{\max }=n$ if and only if all $\varepsilon_{i j}=1$ or $v_{i j}=0$, which is equivalent to having all $a_{i j}=w_{i} / w_{j}$, indicates the consistent situation. Therefore, the problem of deriving the relative weights among criteria in the AHP is equivalent to solving the following mathematical programming problem to obtain $w_{i}$ :

$$
\begin{array}{ll}
\min & \sum_{j=1}^{n}\left\|a_{i j}-\frac{w_{i}}{w_{j}}\right\|_{p} \\
\text { s.t. } & \sum_{i=1}^{n} w_{i}=1, \quad \forall 1 \leq i<j \leq n,
\end{array}
$$

where $\|\cdot\|_{p}$ denotes the $p$-norm and $p \in\{1,2, \ldots\}$. Note that, in this paper, we set $p=2$ in our model.

As mentioned previously, although the AHP is widely used in the field of decision making, it cannot deal with the situation of correlation between criteria. Hence, we propose the extension of the AHP by considering the correlation between criteria in the following section.

\section{Correlated Analytic Hierarchy Process}

In this section, we will propose a way to extend the AHP to consider the correlation between criteria. In order to avoid the confusion between the proposed model and AHP/ANP, we first introduce the following four effects which could happen between criteria as follows.

(1) Outer-dependence $(\rightarrow)$ : a criterion influencing another criterion is called outer-dependence.

(2) Inner-dependence loop $(\hookleftarrow)$ : a criterion has an innerdependence loop if its elements are to depend on each other.

(3) Feedback $(\rightleftarrows)$ : two criteria depending on each other are called feedback.

(4) Correlation $(\leftrightarrow)$ : a criterion correlated to another criterion is called correlation. It should be highlighted that correlation does not imply causation between criteria.

We should highlight that the AHP can account for the outer-dependence effect from upper criteria but claims each subcriterion is independent of the other. Although the ANP can account for the effects of outer-dependence, innerdependence loop, and feedback, it cannot deal with the correlation between criteria.

Let us first consider Figure 3 to describe the presented problem which is considered in this paper.

According to the presentation of Figure 3, it can be seen that Criteria 1 and 2 are considered to affect the decision of the problem. Criterion 1 can be divided into 3 independent subcriteria and so can Criterion 2. We should highlight that since Criteria 1 and 2 are correlated with each other, this problem cannot be solved neither by the AHP nor by the ANP.

In order to consider the correlation effect in the AHP, we should first quantify the correlation matrix between criteria 


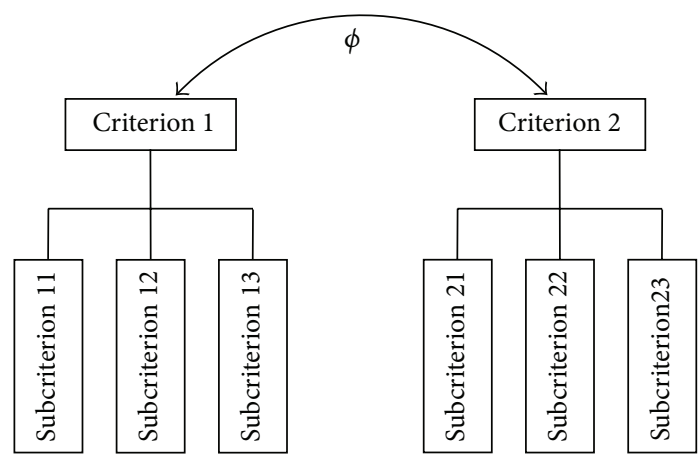

FIGURE 3: The concept of correlation between criteria.

which is given by an expertise. Take Figure 3 as the example, we can obtain the following correlation matrix:

$$
\mathbf{R}=\left[\begin{array}{llllll}
r_{11} & r_{12} & r_{13} & r_{14} & r_{15} & r_{16} \\
r_{21} & r_{22} & r_{23} & r_{24} & r_{25} & r_{26} \\
r_{31} & r_{32} & r_{33} & r_{34} & r_{35} & r_{36} \\
r_{41} & r_{42} & r_{43} & r_{44} & r_{45} & r_{46} \\
r_{51} & r_{52} & r_{53} & r_{54} & r_{55} & r_{56} \\
r_{61} & r_{62} & r_{63} & r_{64} & r_{65} & r_{66}
\end{array}\right] .
$$

Or we can rewrite the above correlation matrix as

$$
\mathbf{R}=\left[\begin{array}{ll}
\mathbf{R}_{11} & \mathbf{R}_{12} \\
\mathbf{R}_{21} & \mathbf{R}_{22}
\end{array}\right]
$$

where

$$
\begin{aligned}
\mathbf{R}_{11}=\left[\begin{array}{lll}
r_{11} & r_{12} & r_{13} \\
r_{21} & r_{22} & r_{23} \\
r_{31} & r_{32} & r_{33}
\end{array}\right], & \mathbf{R}_{12}=\left[\begin{array}{lll}
r_{14} & r_{15} & r_{16} \\
r_{24} & r_{25} & r_{26} \\
r_{34} & r_{35} & r_{36}
\end{array}\right], \\
\mathbf{R}_{21}=\left[\begin{array}{lll}
r_{41} & r_{42} & r_{43} \\
r_{51} & r_{52} & r_{53} \\
r_{61} & r_{62} & r_{63}
\end{array}\right], & \mathbf{R}_{22}=\left[\begin{array}{lll}
r_{44} & r_{45} & r_{46} \\
r_{54} & r_{55} & r_{56} \\
r_{64} & r_{65} & r_{66}
\end{array}\right] .
\end{aligned}
$$

In this example, $\mathbf{R}_{11}=\mathbf{R}_{22}=\mathbf{0}$ since they do not have the self-correlation effect. Note that the self-correlation effect could happen in other situations. In addition, it should be highlighted that $\mathbf{R}_{j i}=\mathbf{R}_{i j}, \forall i, j$, because the correlation effect is symmetric.

Then, we assume that if Criterion $i$ is highly correlated to Criterion $j$, they have similar weights or influence to the problem. Hence, if we obtain the correlation matrix between criteria, we can derive the first objective to maximize the correlation, that is, $\mathbf{w}^{\prime} \mathbf{R} \mathbf{w}$. Then, we incorporate (11) to the proposed model to form the biobjective programming model.

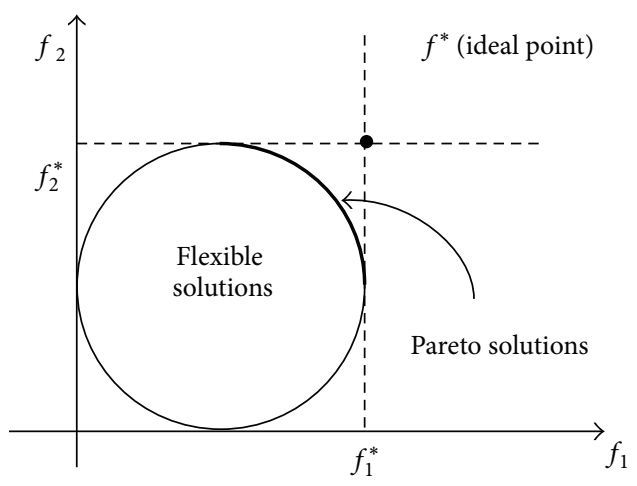

FIGURE 4: Concept of the compromise solutions.

The 2-level correlated analytic hierarchy process (CAHP) can be formulated by the following biobjective programming model:

$$
\begin{array}{ll}
\max & \mathbf{w}^{\prime} \mathbf{R} \mathbf{w}, \\
\min & \left\|a_{I J}-\frac{w_{I}}{w_{J}}\right\|_{p}+\left\|a_{i j}-\frac{w_{i}}{w_{j}}\right\|_{p} \\
\text { s.t. } \quad & \sum_{I=1}^{L} w_{I}=1, \\
& w_{I}=\sum_{i=1}^{k_{i}} w_{i}, \quad I=1, \ldots, L ; i=1, \ldots, l, \\
& w_{I}, w_{i} \in[0,1], \quad \forall I=1, \ldots, L ; i=1, \ldots, l,
\end{array}
$$

where $a_{I J}$ denotes the given estimated weight ratio of the upper-level Criteria $I$ and $J, w_{I}$ denotes the true weight of the upper-level $I$ th criterion, $a_{i j}$ denotes the given estimated weight ratio of the lower-level Criteria $i$ and $j$, and $w_{i}$ denotes the true weight of the lower-level $i$ th criterion. In addition, the upper-level $I$ th criterion can be divided into $k_{i}$ lower-level criteria.

In order to solve the above biobjective programming, we introduce the compromise solution as follows. In a multiple objective programming (MOP) problem, an ideal (or utopia) point is usually not attainable while trade-offs between objectives exist. Hence, $\mathrm{Yu}$ [10] proposed the compromise solutions to determine the optimal solution, which is closest to the ideal point, among Pareto solutions based on the $L_{p}$ norm distance. The concept of the compromise solutions can be depicted as shown in Figure 4.

The $L_{p}$-norm distance between a point and the ideal point can be defined as

$$
d_{p}=\left\|f^{*}-f\right\|_{p}, \quad p=1, \ldots, \infty .
$$

In a generalized optimal problem, the distance measured by the $L_{p}$-norm between a point and the ideal point can be presented as shown in Figure 5. The left-lower square belongs to the maximized problems (maximize all the objective functions) and is the case dealt with in this section. From 


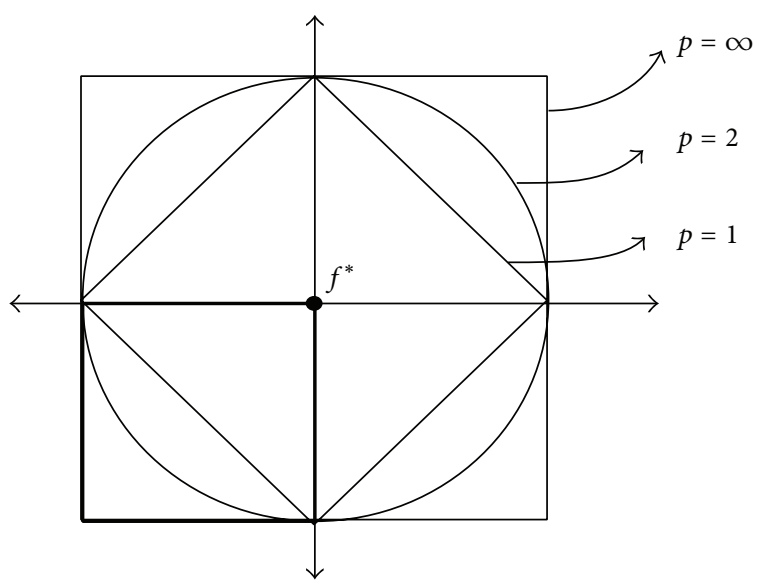

Figure 5: Concept of the $L_{p}$-norm distance.

Figure 5, it can be seen that the shape of $p=1$ is a square diamond, $p=2$ is a circle, and $p=\infty$ is a square. The different shapes of the $L_{p}$-norm may result in the different result of the optimal solution. In addition, other kinds of the $L_{p}$-norm are less discussed because they have no concrete meaning in practice.

The procedures of the compromise solutions can be demonstrated by considering a multiple objective programming (MOP) problem as follows:

$$
\begin{array}{ll}
\max & \mathbf{z}(\mathbf{x})=\left[z_{1}(\mathbf{x}), z_{2}(\mathbf{x}), \ldots, z_{n}(\mathbf{x})\right] \\
\text { s.t. } & g(\mathbf{x}) \leq \mathbf{b} \\
& \mathbf{x} \geq \mathbf{0}
\end{array}
$$

The first step of the compromise solutions is to determine the ideal point of each objective. This can be done by optimizing each objective as follows:

$$
\begin{array}{ll}
\max & z_{j}(\mathbf{x}) \\
\text { s.t. } & (\mathbf{x}) \leq \mathbf{b}, \\
& \mathbf{x} \geq \mathbf{0} .
\end{array}
$$

Then, we can obtain the ideal point as $\mathbf{z}^{*}=\left(z_{1}^{*}, z_{2}^{*}, \ldots, z_{n}^{*}\right)$. Next, we want to determine which point located on the Pareto solutions is closest to the ideal point as the optimal solution. Hence, we can use the concept of the $L_{p}$-norm to measure the distance between objective values and the ideal point and formulate the compromise solution method as [10]

$$
\begin{array}{ll}
\min & d_{p}=\left\{\sum_{j=1}^{n} w_{j}^{p}\left[z_{j}^{*}(\mathbf{x})-z_{j}(\mathbf{x})\right]^{p}\right\}^{1 / p}, \quad p=1, \ldots, \infty \\
\text { s.t. } & g(\mathbf{x}) \leq \mathbf{b}, \\
& \mathbf{x} \geq \mathbf{0},
\end{array}
$$

where $w_{j}$ denotes the importance of the $j$ th objective. Besides using the traditional $L_{p}$-norm, Gershon and Duckstein [11]

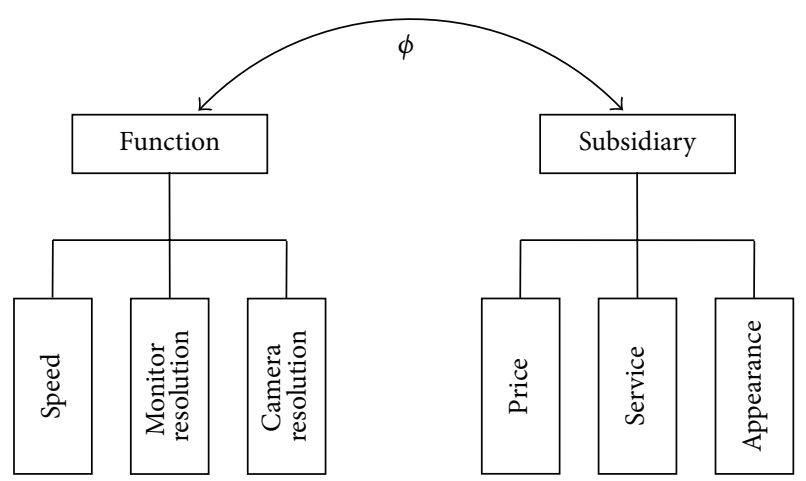

FIgURE 6: The correlated hierarchical structure of the problem.

proposed the normalized $L_{p}$-norm and formulated the compromise solutions as

$$
\begin{array}{ll}
\min & d_{p}=\left\{\sum_{j=1}^{n} w_{j}^{p}\left[\frac{z_{j}^{*}(\mathbf{x})-z_{j}(\mathbf{x})}{z_{j}^{*}(\mathbf{x})-z_{j}^{-}(\mathbf{x})}\right]^{p}\right\}^{1 / p}, \\
& p=1, \ldots, \infty \\
\text { s.t. } & g(\mathbf{x}) \leq \mathbf{b}, \\
& \mathbf{x} \geq \mathbf{0},
\end{array}
$$

where $z_{j}^{-}(\mathbf{x})$ denotes the minimum value of the $j$ th goal. In this paper, we adopt Duckstein's method and transform the first objective to minimization by multiply -1 . Next, we propose a numerical example to demonstrate the proposed model as follows.

\section{A Numerical Example}

Let us consider a purchasing decision of a smart phone to illustrate the proposed method as follows. Assume that two criteria, for example, Function and Subsidiary, are considered to determine a smart phone. Function can be divided into three subcriteria, Speed, Monitor Resolution, and Camera Resolution. On the other hand, Subsidiary can be divided into Price, Service, and Appearance. In addition, Function and Subsidiary are considered to be correlated with each other. Hence, the hierarchical structure of the problem can be shown as in Figure 6.

Next, we assume that Criterion 1 is two times more important than Criterion 2 and consider the following comparison matrices of subcriteria:

$$
\begin{gathered}
\mathbf{W}_{11}=\left[\begin{array}{ccc}
1 & 2 & 3 \\
\frac{1}{2} & 1 & 5 \\
\frac{1}{3} & \frac{1}{5} & 1
\end{array}\right], \quad \mathbf{W}_{22}=\left[\begin{array}{ccc}
1 & \frac{1}{3} & \frac{1}{4} \\
3 & 1 & \frac{1}{2} \\
4 & 2 & 1
\end{array}\right], \\
\mathbf{W}_{12}=\mathbf{W}_{12}=\mathbf{0 .}
\end{gathered}
$$

Then, we can give the correlation matrices as follows:

$$
\mathbf{R}_{12}=\mathbf{R}_{21}=\left[\begin{array}{lll}
0.2 & 0.3 & 0.2 \\
0.4 & 0.5 & 0.3 \\
0.2 & 0.4 & 0.3
\end{array}\right], \quad \mathbf{R}_{11}=\mathbf{R}_{22}=\mathbf{0} .
$$


TABLE 1: The comparison of the weight between the AHP and CAHP.

\begin{tabular}{lcccccccc}
\hline Method & $w_{1}$ & $w_{2}$ & $w_{11}$ & $w_{12}$ & $w_{13}$ & $w_{21}$ & $w_{22}$ & $w_{23}$ \\
\hline AHP & 0.6667 & 0.3333 & 0.2594 & 0.3341 & 0.0732 & 0.0422 & 0.1144 & 0.1767 \\
CAHP & 0.6820 & 0.3180 & 0.2667 & 0.3405 & 0.0748 & 0.0403 & 0.1083 & 0.1694 \\
\hline
\end{tabular}

TABLE 2: The stability analysis of the proposed method.

\begin{tabular}{lcccccccc}
\hline$\Delta$ & $w_{1}$ & $w_{2}$ & $w_{11}$ & $w_{12}$ & $w_{13}$ & $w_{21}$ & $w_{22}$ & $w_{23}$ \\
\hline$\Delta=0$ & 0.6820 & 0.3180 & 0.2667 & 0.3405 & 0.0748 & 0.0403 & 0.1083 & 0.1694 \\
$\Delta=10$ & 0.6813 & 0.3187 & 0.2660 & 0.3406 & 0.0747 & 0.0404 & 0.1085 & 0.1698 \\
$\Delta=20$ & 0.6831 & 0.3169 & 0.2673 & 0.3409 & 0.0749 & 0.0402 & 0.1076 \\
$\Delta=30$ & 0.6777 & 0.3223 & 0.2640 & 0.3395 & 0.0743 & 0.0407 & 0.1111 & 0.1704 \\
\hline
\end{tabular}

Next, we can employ (15) to derive the result of the CAHP by solving the biobjective programming as follows:

$$
\begin{gathered}
\min -\left(0.2 w_{11} w_{21}+0.3 w_{11} w_{22}+0.2 w_{11} w_{23}+0.4 w_{12} w_{21}\right. \\
+0.5 w_{12} w_{22}+0.3 w_{12} w_{23}+0.2 w_{13} w_{21} \\
\left.+0.4 w_{13} w_{22}+0.3 w_{13} w_{23}\right) \\
\min \left\|\left(2-\frac{w_{1}}{w_{2}}\right)+\left(\frac{1}{2}-\frac{w_{2}}{w_{1}}\right)\right\| \\
+\left\|\left(2-\frac{w_{11}}{w_{12}}\right)+\cdots+\left(2-\frac{w_{23}}{w_{22}}\right)\right\|
\end{gathered}
$$

s.t. $w_{1}+w_{2}=1$,

$$
w_{1}=w_{11}+w_{12}+w_{13}
$$

$$
\begin{aligned}
& w_{2}=w_{21}+w_{22}+w_{23} \\
& w_{1}, w_{2}, w_{11}, w_{12}, w_{13}, w_{21}, w_{22}, w_{23} \geq 0
\end{aligned}
$$

Then, we can use the compromise solution to solve the above problem and derive the result of the CANP. In addition, if we take off the first objective, we can derive the result of the traditional AHP. The results of the AHP and CAHP can be presented as shown in Table 1.

The result of CAHP is slightly different from that of the AHP due to the correlation effect between criteria. In order to understand the stability of the proposed method, we add some deviations, that is, random errors, into the previous correlation matrix such that

$$
\mathbf{R}_{12}=\mathbf{R}_{21}=\left[\begin{array}{lll}
0.2+\delta_{1} \times 0.2 \times \Delta \% & 0.3+\delta_{2} \times 0.3 \times \Delta \% & 0.2+\delta_{3} \times 0.2 \times \Delta \% \\
0.4+\delta_{4} \times 0.4 \times \Delta \% & 0.5+\delta_{5} \times 0.5 \times \Delta \% & 0.3+\delta_{6} \times 0.3 \times \Delta \% \\
0.2+\delta_{7} \times 0.2 \times \Delta \% & 0.4+\delta_{8} \times 0.4 \times \Delta \% & 0.3+\delta_{9} \times 0.3 \times \Delta \%
\end{array}\right]
$$

where $\delta_{i}, i=1, \ldots, 9$, is a random dummy variable denoted by $\{-1,1\}$ and $\Delta$ is a user-defined value ranging from $[0,100]$ to indicate the possible fluctuation. For example, we specify $\Delta=10$ and run $\delta_{i}$ once to obtain the correlation matrix as

$$
\mathbf{R}_{12}=\mathbf{R}_{21}=\left[\begin{array}{lll}
0.2-1 \times 0.2 \times 10 \% & 0.3+1 \times 0.3 \times 10 \% & 0.2+1 \times 0.2 \times 10 \% \\
0.4+1 \times 0.4 \times 10 \% & 0.5-1 \times 0.5 \times 10 \% & 0.3-1 \times 0.3 \times 10 \% \\
0.2-1 \times 0.2 \times 10 \% & 0.4+1 \times 0.4 \times 10 \% & 0.3-1 \times 0.3 \times 10 \%
\end{array}\right]
$$

Then, we can simulate 3 times the stability analysis of the proposed method with respect to different $\Delta$ values and compare these results with the original solution as shown in Table 2.

From the results of Table 2, it can be shown that the proposed method presents the excellent stability and prevents the rank reversal of the criteria here; even the fluctuation or expert's error is equal to $30 \%$.
Next, we modify the previous original correlation matrix as follows:

$$
\mathbf{R}_{12}=\mathbf{R}_{21}=\left[\begin{array}{lll}
0.7 & 0.4 & 0.5 \\
0.6 & 0.7 & 0.8 \\
0.8 & 0.6 & 0.9
\end{array}\right] .
$$

With the same procedure above, we can obtain the modified result of the CAHP, as shown in Table 3. 
TABLE 3: The modified result of the CAHP.

\begin{tabular}{|c|c|c|c|c|c|c|c|c|}
\hline Method & $w_{1}$ & $w_{2}$ & $w_{11}$ & $w_{12}$ & $w_{13}$ & $w_{21}$ & $w_{22}$ & $w_{23}$ \\
\hline CAHP & 0.7175 & 0.2825 & 0.2834 & 0.3556 & 0.0785 & 0.0357 & 0.0980 & 0.1488 \\
\hline
\end{tabular}

From Table 3, it can be seen that the final result may change depending on the specific correlation matrix. However, the traditional AHP can only obtain the same result, since it ignores the correlation between criteria. Next, we propose the discussions in the following section according to the finding of the numerical example.

\section{Discussions}

The AHP/ANP has been the most popular tool for decision making in the recent 20 years, because of its simplicity and reasonability. The AHP is used when we consider the situation of outer-dependence between independent criteria. On the other hand, the ANP can account for the effects of outerdependence, inner-dependence, and feedback in a network structure. However, the traditional AHP/ANP ignores the situation of correlation between criteria which indicates that one criterion is correlated to another. In realistic situation, the correlation between criteria is common and important. It may provide information for a decision maker to derive more accurate result.

In this paper, the CAHP is proposed to consider the correlation effect between criteria in the AHP. First, we ask responders to quantify the correlation matrix. Then, we consider a biobjective programming model to derive the weights of criteria. The first objective incorporates the information of correlation between criteria and maximizes $\mathbf{w}^{\prime} \mathbf{R} \mathbf{w}$, where $\mathbf{w}$ and $\mathbf{R}$ denote the weight vector and correlation matrix, respectively. The philosophy above is to claim that the weights of two criteria are similar when they have higher degree of correlation.

The second objective follows the purpose of the AHP to minimize the error between the given and true weight ratios between criteria. Finally, we use the compromise solution to deal with the model and obtain the final result. It should be highlighted that the AHP can be considered as the special case of the proposed model when the values of correlation between criteria are zero. Then, the biobjective programming is reduced to single-objective programming.

\section{Conclusions}

In this paper, we extend the AHP to consider the correlation effect between criteria. Then, a biobjective programming model is developed to derive the result of the CAHP. In addition, we use the compromise solution to solve the biobjective programming model. From the result of the numerical example, it can be seen that the correlation effect between criteria can be incorporated into the traditional AHP. The proposed model can be reduced into the AHP when we take off the first objective, that is, ignore correlation between criteria.

\section{Conflict of Interests}

The authors declare that there is no conflict of interests regarding the publication of this paper.

\section{References}

[1] D. Bernoulli, "Specimen Theoriae Novae de Mensura Sortis," Commentarri Academiae Scientiarum Imperialis Petropolitanae, vol. 5, pp. 175-192, 1738.

[2] J. von Neumann and O. Morgenstern, Theory of Games and Economic Behavior, Princeton, NJ, USA, Princeton University Press, 2nd edition, 1947.

[3] D. Dubois and H. Prade, Fuzzy Sets and Systems, Academic Press, New York, NY, USA, 1980.

[4] T. L. Saaty, "A scaling method for priorities in hierarchical structures," Journal of Mathematical Psychology, vol. 15, no. 3, pp. 234-281, 1977.

[5] T. L. Saaty, The Analytic Hierarchy Process, McGraw-Hill, New York, NY, USA, 1980.

[6] P. L. Yu, Multiple Objective Decision Making: Concepts, Techniques and Extension, Plenum Press, New York, NY, USA, 1985.

[7] J. J. Huang, "A matrix method for the fuzzy analytic hierarchy process," International Journal of Uncertainty, Fuzziness and Knowledge-Based Systems, vol. 19, no. 2, pp. 401-414, 2011.

[8] T. L. Saaty, Decision Making with Dependence and Feedback, The Analytic Network Process, RWS Publications, Pittsburgh, Pennsylvania, 1996.

[9] T. L. Saaty, "Decision-making with the AHP: why is the principal eigenvector necessary," European Journal of Operational Research, vol. 145, no. 1, pp. 85-91, 2003.

[10] P. L. Yu, "A class of solutions for group decision problems," Management Science, vol. 19, pp. 936-946, 1973.

[11] M. Gershon and L. Duckstein, "A procedure for selection of a multiobjective technique with application to water and mineral resources," Applied Mathematics and Computation, vol. 14, no. 3, pp. 245-271, 1984. 


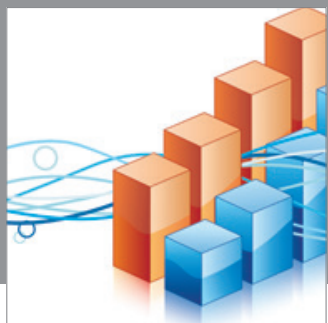

Advances in

Operations Research

mansans

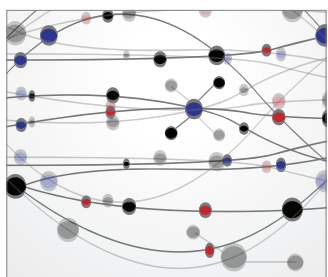

The Scientific World Journal
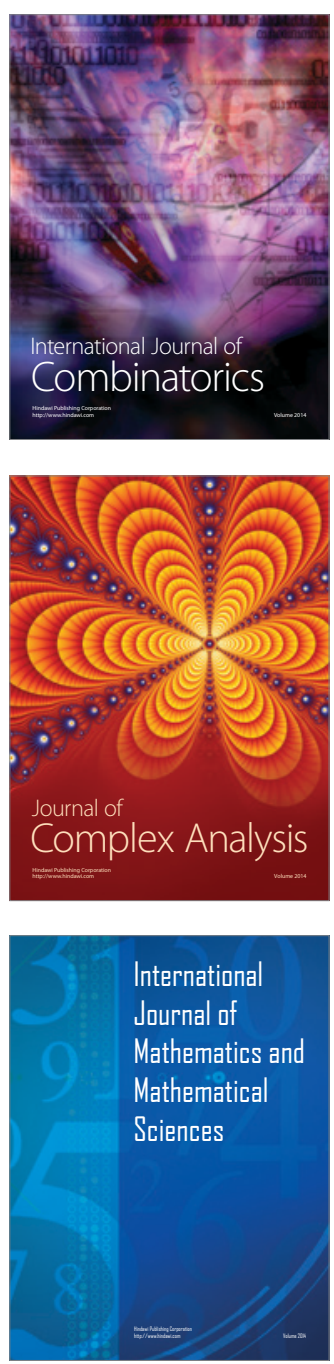
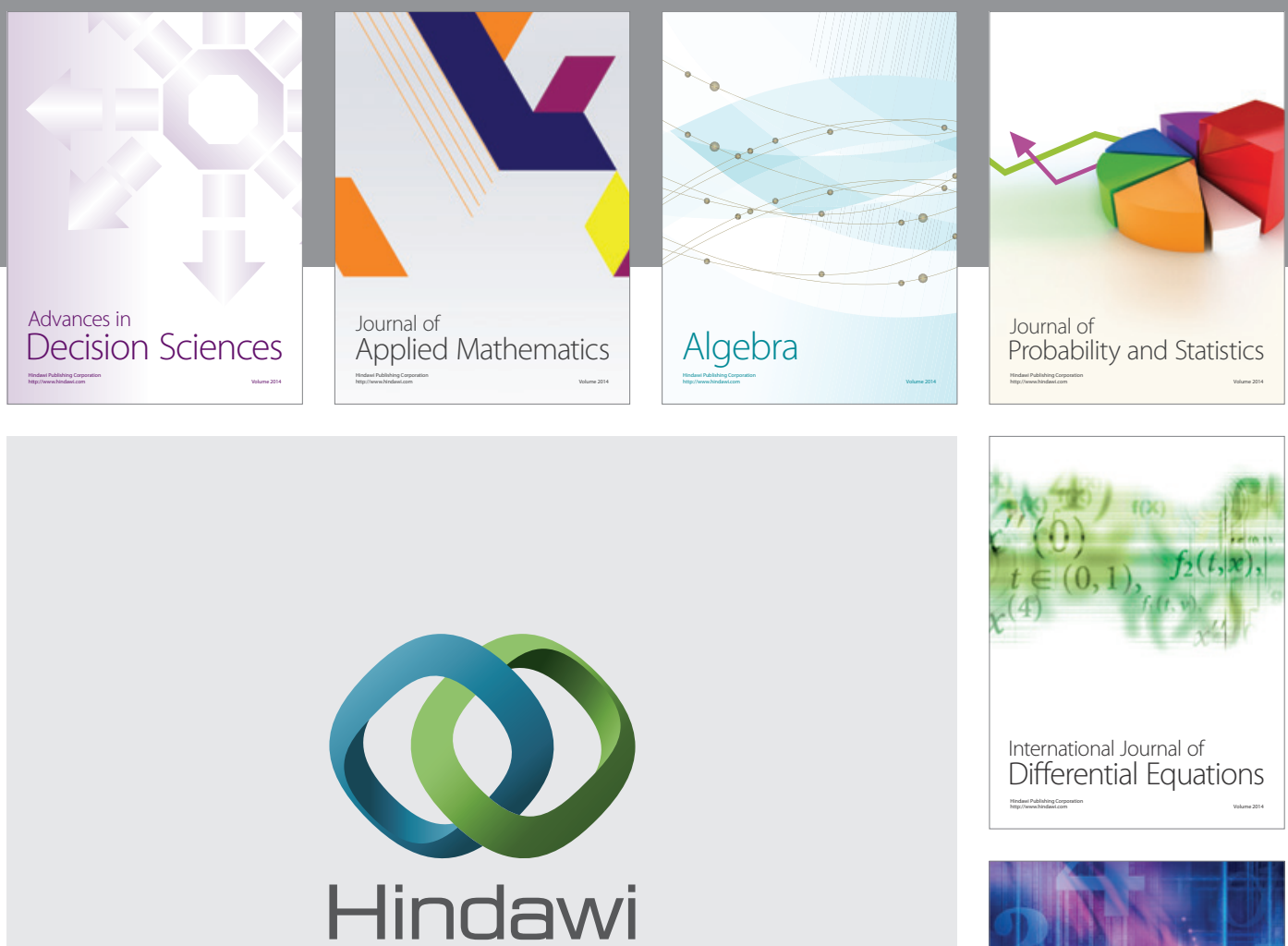

Submit your manuscripts at http://www.hindawi.com
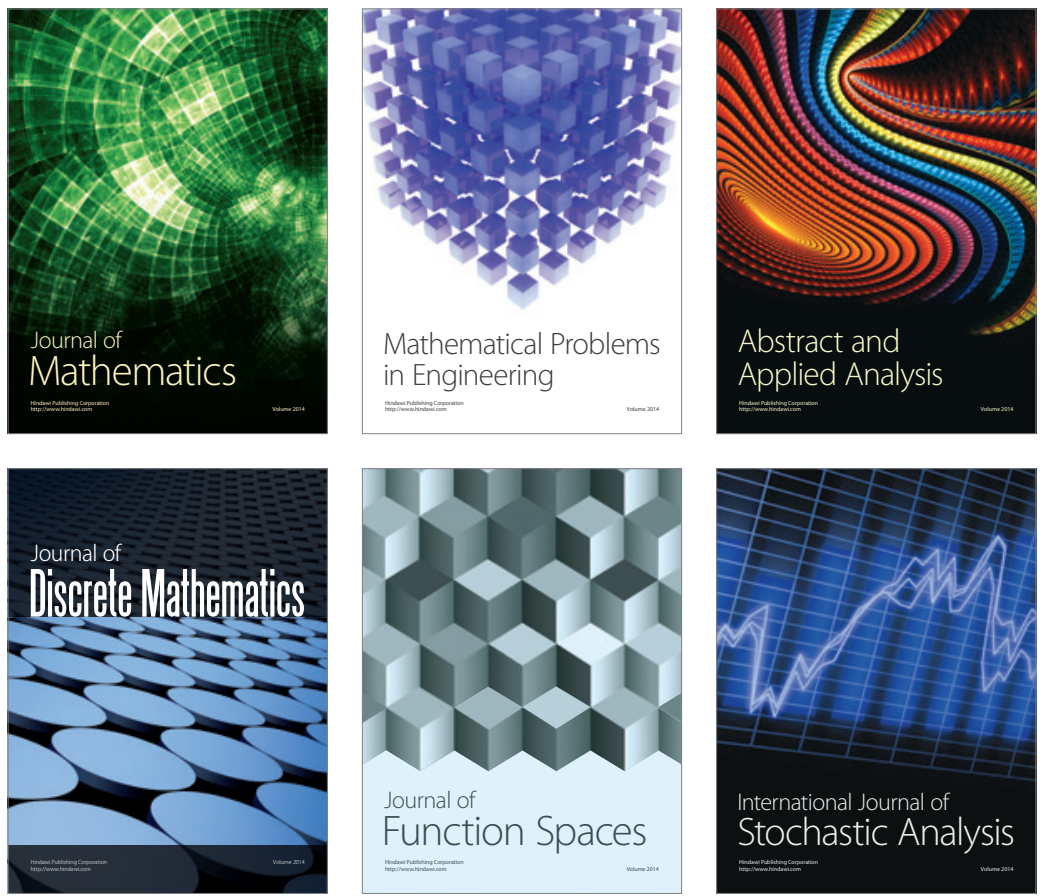

Journal of

Function Spaces

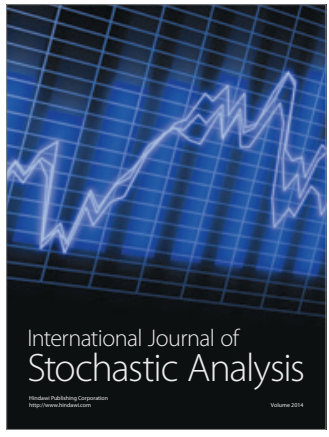

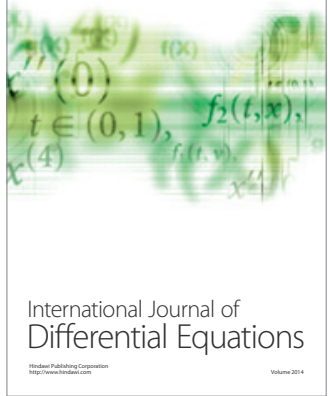
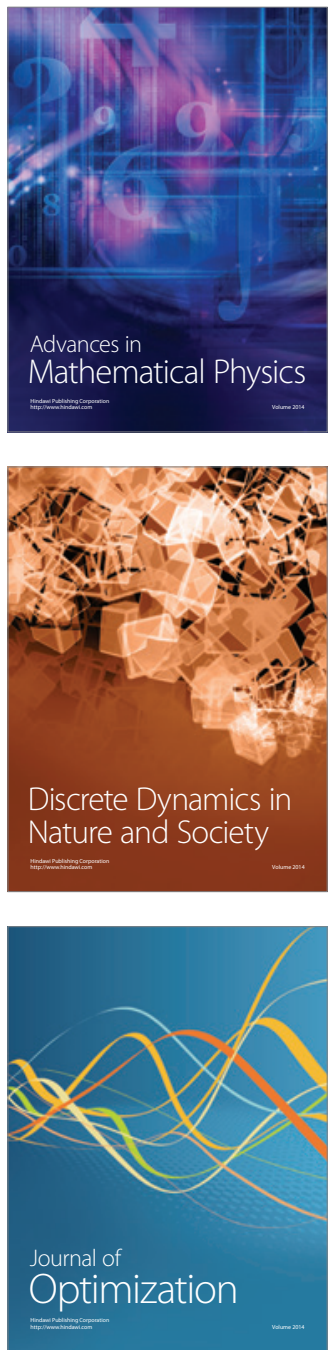